\title{
Redefining dental care to serve patients with special health care needs: A review
}

\author{
Yogeshwari Krishnan",, Lakshya Vishnoi², Himanshu Aeran³, ${ }^{1,}$ arun Kumar ${ }^{4}$ \\ ${ }^{1}$ Intern, ${ }^{2}$ BDS Student, ${ }^{3}$ Director Principal, Professor \& HOD, ${ }^{4}$ Professor, ${ }^{3,4}$ Dept. of Prosthodontics, Seema Dental College and \\ Hospital, Rishikesh, Uttrakhand, India
}

*Corresponding Author:

Email: dryogeshwari1@gmail.com

\begin{abstract}
Some of the most wonderful people are those who, cannot fit into a mould and so are the patients with special needs. The patients require medical, physical, psychological, or social situations that make it necessary for us to modify our normal dental routines in order to provide dental treatment for such individuals. These individuals include, but are not just limited to the people with developmental disabilities, complex medical problems, and significant physical limitations ${ }^{1,2}$ alone. The population of these patients are reported to have higher rates of poor oral hygiene and thus having a greater incidence of gingivitis and periodontitis, and also dental caries, than for the others, falling in the mould of general population. This review article aims at describing various measures that can be taken for providing care to such individuals.
\end{abstract}

Keywords: Special needs, Special Dental Care, Physical disorders, Developmental disorders, Mental disorders, Oral health care needs.

\section{Introduction}

It is a big basket of conditions which fall under the term "special needs" so to begin with talking on dealing with such patients we must first know who all they can be. Patients with special needs are the patients with physical, medical, mental or social situations that make it necessary for us to modify normal dental routines in order to provide dental treatment for such individuals. The AAPD defines special health care needs as "any physical, developmental, mental, sensory, behavioural, cognitive, or emotional impairment or limiting condition that requires medical management, health care intervention, and/or use of specialized services or programs. The condition may be congenital, developmental, or acquired through disease, trauma, or environ- mental cause and may impose limitations in performing daily self-maintenance activities or substantial limitations in a major life activity. Health care for individuals with special needs requires specialized knowledge acquired by additional training, as well as increased awareness and attention, adaptation, and accommodative measures beyond what are considered routine."3

A special needs patient also encompasses the patients who are elderly and/or suffering from mobility issues, those who are immunocompromised, having medical conditions like epilepsy, bleeding disorders, diabetes, cardiovascular diseases or any other systemic illnesses which require changes in the treatment protocol. In such conditions the patient's physician must also be consulted. Even a pregnant female is considered as one with special needs. ${ }^{4}$

This category majorly includes those who are mentally ill, that is from having mild to severe mental retardation to developmental disorders, cerebral palsy, autism, Asperger's Syndrome, Downs Syndrome, psychosis etc. A strong interaction is known to exist between mental health and oral health. Even burning mouth syndrome can be associated with patients getting treated with depression or anxiety. Consideration should be given to patients with eating disorders which can be seen as erosion or the palatal surfaces and in mood disorders attrition is common but in people with bipolar disorders it is common to see other issues like that of overzealous brushing or flossing hence causing abrasion.

Oral health is an important part of physical health. As correctly quoted at places that "mouth is the mirror of the body as eyes are to the soul". Poor oral health is linked to many systemic diseases such as coronary heart disease, bacterial endocarditis, respiratory diseases etc. Oral health also affects the eating, speech and the social and psychological life of the patient. The most common two problems of poor oral health are dental caries and periodontitis resulting in the loss of teeth which may involve the whole dentition and at last may cause complete edentulousness.

Till now the patients with special needs have not shown any improvement in oral health of the general population and thus their special needs must be focused onto and catered.

\section{Categories of patients who have special needs}

For patients with mental disorders: In most of the mental health related conditions the person himself isn't able to take care of his basic needs, daily routine etc. and thus it is the responsibility of the dental professional to guide and educate the care takers so that they seek out and maintain the patient's dental health. Their medications also have a negative impact on their 
oral condition, causing xerostomia to caries specially, gingivitis and periodontitis etc. which are mostly seen in patients with severe mental illness like dementia, schizophrenia. In such cases, the dental treatment involves ensuring a good oral health by involvement of their care takers and scheduling appointments when there is best psychiatric balance for the patient and also keeping appointments short[5].They specifically need to be accompanied to the operatory by their care takers and need for physical restrains etc. might be required. The dentist must also be skilled to deal with children having Communication spectrum disorders like autism, downs syndrome and cerebral palsy and what his tone of voice should be and how he must explain things better to the patient as to what he is doing and always keep finger guards for himself as they mostly have a habit of sudden closure of mouth. The dentist must keep giving the patients rest and plan out small sessions for him. How to segregate patients of varying levels of mental capabilities as accordingly we can know how well the patient would be able to follow the instructions in the operatory and home.

Malocclusions are also bound to occur in patients with developmental disorders including intraoral and perioral muscular abnormalities, delayed tooth eruption, underdevelopment of the maxilla and other oral habits like bruxism and tongue thrusting. Orthodontic treatment might not be an option for all but daily ability of the caregiver to maintain a good oral hygiene is a must. ${ }^{6}$

There must be a closer collaboration between the health clinicians and the dentist to remove the barriers to care, if these are psychosocial or financial. This can go the other way round as well as the dentist may be the first clinician to diagnose a patient of eating disorder. Reluctance of some patients to go for psychiatric treatment, the dentist must council the patient for that too.

Behavioural problems can also complicate oral health care. The anxiety and the fear about the dental treatment may cause some patients to become uncooperative. Their behaviours range from fidgeting or temper tantrums to violent self-injurious behaviour such as head banging, thus making it even more challenging.

The following strategies might help which are:

1. Setting up a perfect dental visit for the patient by the involvement of the whole of the dental team, from receptionist's friendly behaviour to dental assistants caring attitude in the operatory.

2. Doing desensitization programs for the patient and caretakers.

3. Using least restrictions to gain cooperation.

4. Keeping appointments short.

5. Use of immobilization techniques/physical restraints only when it isabsolutely necessary.
6. The dentist should do education programs for care takers mainly and tell them about the need for dental visits and basically how he can help in the problems being faced. Like he can use artificial salivary products for the patient with xerostomia and also do fluoride application for the prevention of further caries. He must educate the patient and the care giver about the nutritional intake and the type of diet which would keep his oral and systemic health in better shape. ${ }^{7}$

For patients with mobility issues dental care must be provided to them at home but this isn't mostly achieved easily due to poor facilities, lack to skills, and lack of daily support from care takers. For others the dentist needs to observe the impact of the disability and look for challenges such as uncontrolled body movements, posture and to maintain a clear path of movement throughout the treatment. Also if needed to transfer the patient to the dental chair from a wheel chair the care giver may be asked to help in doing so.Patients who cannot be moved from their wheel chair must be treated while on them.

For those who are visually impaired the dentist must determine the level of assistance required to move about in the clinic, using patients other senses to connect and to establish trust with them and to always face the patient while talking and to tell them about the next step making verbal communication very important.

For the patients who have hearing defects thepatient must be first asked to adjust his hearing aids or to turn them off since the sounds of some instruments may be too disturbing for them, If he is able to read lips, the dentist may talk in a slow and calm way. If he speaks and understands sign language ask him to get his family or friend who can act as an interpreter. Visual feedback in such cases is of great importance, and maintaining eye contact is a must.

Patients with allergies also fall into this basket. Latex allergy is a serious problem and for patients who have just undergone a surgery or have spina bifida are more prone to it. We must take a proper medical history at first about it, if this is known to happen to a patient and when and how did it happen and about what the outcome may be like. Their appointment must also be given early during the day as then the air in the operatory is also free of allergens and also the clinician must use latex free gloves.

Patients with developmental or acquired orofacial conditions: ${ }^{8}$ The oral health care needs of patients with developmental or acquired orofacial conditions require special considerations. While these individuals usually require shorter appointments and advanced behaviour guidance techniques which are commonly associated with children having Special Health Care Needs, management of their oral conditions presents other unique challenges. Developmental defects such as hereditary ectodermal dysplasia, where most teeth are 
missing or malformed, cause lifetime problems that can be devastating for the children and adults. ${ }^{5}$ Starting from the first contact with the child and his family, every effort must be made to assist the family in adjusting to and understanding to the complexity of the condition and to its related oral needs. ${ }^{9}$

The dental practitioner must be sensitive to the psychosocial well-being of the patient, as well as the effects of the condition on his growth, function, and appearance. Congenital oral conditions may require therapeutic intervention which should be timed to coincide with the developmental milestones. Patients with conditions such as ectodermal dysplasia, epidermolysisbullosa, cleft lip/palate, and oral cancer frequently require an interdisciplinary team approach for their care. Coordinating delivery of services by the various health care providers can be crucial for successful treatment outcomes. ${ }^{10}$

For children with hereditary hypodontia and/or oligodontia, removable or fixed prostheses (including complete dentures or over-dentures) and/or implants may be indicated. ${ }^{11}$ To be done only after studying and knowing about their other medical needs.

To care better for the patients with special needs

The following points should be considered:

First dental visit $\&$ scheduling of appointments: The care taker's and the patient's initial contact with the dental Setup gives both parties an opportunity to address the patient's primary oral health care needs and to confirm the appropriateness of scheduling an appointment with that particular practitioner. Along with the patient's name, age, and chief complaint. The receptionist should determine the presence and nature of any Special needs and also the names of the patient's medical care providers. The office staff, under the guidance of the dentist, should determine the length of the appointment. The need for increasing dentists and his team, time as well as customized services should be documented so the office staff is prepared to accommodate the patient at each following visit. ${ }^{12}$

When scheduling appointments for patients with Special Health Care Needs, it is imperative that the dentist be familiar with and comply with Health Insurance Portability and Accountability Act (HIPAA) and AwDA regulations applicable to dental practices.[13] HIPAA is meant to protect the patient's privacy and AwDAto prevent any discrimination on the basis of disability.

Working together: This may involve an extra assistant, or a person the patient trusts who can relay information and facilitate better communication and understanding, so that things function smoothly. This also involves the collaboration with the patients' medical health care provider for history and any emergency condition. ${ }^{4}$

Assessing the patient: Familiarity with the patient's medical history is essential to decrease the risk of aggravating a medical condition while providing with dental care. An accurate, comprehensive, and up-todate medical history is a necessity for correct diagnosis and effective treatment planning. Information regarding the chief complaint, history of present illness, medical conditions, medical care providers, hospitalizations, anaesthetic experiences, current medications, allergens, immunization status, a complete review of all body systems, family and social histories, and a thorough dental history should be obtained from the patient and his care givers. ${ }^{14}$

If the patient/his caregivers are unable to provide accurate information, consultation with the patient's physician may be required. At each patient visit, his history should be consulted with his physician and kept updated. Changes in medications, recent medical attention for illness or any injury, newly diagnosed medical conditions, need to be documented.

A preventive program depending on case, including call schedule, should be recommended after evaluation of the patient's caries risk assessment, oral health needs, and abilities. A summary of the oral findings and specific treatment recommendations should be provided to the patient and parent/ caregiver. When appropriate, the physicians, nurses or social workers working for the patients care should be informed of any significant findings.

Medical considerations: ${ }^{6}$ The patient's physician should be consulted regarding his medications, sedation, anaesthesia, and special restrictions .For preparing to ensure the safe delivery of dental care to the patient. The dentist and his staff should always be prepared to manage every type of medical emergency.

Communication: Communication is the key as in any other treatment. Management beginning with general assessment for needs by using standard checklists and spreading awareness among masses. With special education programs to be done for the care givers of such patients and thus teaching them the regular must dos' and donts' to achieve better oral health care like how to brush and what mouthwash must be used etc. Also telling them the use of visiting the dentist and what he can do, example he could help patients with dry mouth/xerostomia which is caused by various kinds of drugs, by giving artificial salivary supplements to the patient and also by application of fluoride to prevent caries and also professional cleaning of teeth so that the chances for other problems like gingivitis and periodontitis is also reduced and of course about the treatment required depending on person to person and their diagnosed conditions Also, better facilities must be available for giving home visits and treating the patients at their residence for those who have severe mobility problems and also giving training programs to dental professionals during their dental school so that these skills of managing and pertaining to the needs of these special patients can be carried on with. 
Care givers are our valuable resource while treating patients with special needs because they provide valuable information and provide feedback on ideal time of day for the treatment, behaviour management, communication assistance, the treatment needs and additional information about patient's disabilities. ${ }^{15}$

Pre-treatment planning is a must and should include a thorough review of the patient's medical history. Appropriate intervals for washroom breaks, and desensitizing the patient before the appointment with a tell-show-do technique aims at reducing the anxiety level.

While working an attempt should be made to communicate directly with the patient during the provision of dental care. The patient who cannot communicate verbally may communicate in a variety of other ways. A parent, family member, or caretaker may need to be present in the operatory, to facilitate better communication and/or to provide information that the patient cannot provide. ${ }^{16}$

Consent: Before starting the treatment the patient must give his signed consent known as informed consent or the caregivers of the patient must be asked to sign proxy consent on behalf of the patient in case he, himself cannot.A clear information must be given to him including the nature of work, risk, and benefits, recommended treatment modalities, any alternatives to the procedure,the need of any type of anaesthetics, premedication, restrains and possible complications associated with our proposed treatment. All patients must be able to provide a signed consent for dental treatment or have someone present who legally can provide it on their behalf. Informed consent/ assent must be as per, the state laws and it should be well documented in the dental records through a signed and witnessed form. ${ }^{17}$

Behavior guidance and management: Behaviour of such patients can turn out to be very challenging. Because of their dental anxiety or a lack of understanding of dental care, patient's with disabilities may be very resistant. These behaviors can thus interfere with the safe and proper delivery of dental treatment to them.

With the parent/caregiver's assistance, most patients with physical and mental disabilities can be managed in the dental office very nicely. Protective stabilization can be helpful in patients on whom the conventional behaviour guidance techniques do not work. ${ }^{18}$ When protective stabilization is not feasible or effective, sedation or general anaesthesia is the treatment of choice. When this too, is ineffective, an out-patient surgical care facility might be necessary.

Usually a need for stabilization of the patients head can be accomplished by the use of pillows, towels or rolled blankets. The patients head must be raised sufficiently, but within the comforts of the patient to avoid any respiratory compromise. During treatment, oral stabilization via mouth props with rubber or
Styrofoam bite blocks with floss tied through one end to be removed quickly to prevent aspiration, tongue depressors, cotton rolls and rolled gauges can be used.

So to meet the special needs of special patients the dentist must himself be trained and then train others for the benefits of use of the required special dental treatment.

Preventive measures: Patients with special needs are at an increased risk for oral diseases, due to their neglected oral health care as discussed. The patients and their caregivers must be educated well about the concern, as any oral condition later or a progression of the one present would just increase the patients discomfort further. So it's best for them to visit a dentist regularly. So that he can guide them about the patients oral condition and oral hygiene measures to be practiced, which may be simple and effective and would improve the patients oral health. Some of them may be:

1. Brushing with a fluoridated dentifrice twice daily should be emphasized on, to help prevent caries and gingivitis. If patient's sensory issues cause the taste or texture of fluoridated toothpaste to be intolerable, a fluoridated mouth rinse may be applied with the help of a toothbrush. Toothbrushes can also be modified for patients with physical disabilities to brush their own teeth. Electric/motored toothbrushes and floss holders may improve patient compliance. The dentist can also do Fluoride applications to prevent carries.

2. A non-cariogenic diet should be discussed for long term prevention of dental disease. ${ }^{19}$ When a diet rich in carbohydrates seems medically necessary (eg., for weight gain), the dentist should provide strategies to decrease the caries risk by altering frequency of and/or increasing preventive measures. As well as, other oral side effects such as xerostomia, gingival overgrowth from medications should be reviewed.

3. Patients may benefit from sealers. Sealants reduce the risk of caries in susceptible pits and fissures of primary and permanent teeth. ${ }^{20}$ Topical fluorides may be indicated when caries risk is increased. ${ }^{21}$ Interim therapeutic restoration (ITR), ${ }^{22}$ using materials such as glass ionomers that release fluoride, may be useful as both preventive and therapeutic approaches in such patients.

4. For cases of gingivitis and periodontal diseases, chlorhexidine mouth rinses may be useful. The patients who might swallow a rinse, toothbrush can be used to apply the chlorhexidine mouthwash onto their teeth. Patients having severe dental diseases may need to be seen every two to three months by the specialists like a Periodontist in the case of severe chronic periodontitis.

5. Preventive strategies should also include traumatic injuries, Including anticipatory guidance about the risk of trauma eg in patients with seizure disorders 
or motor skills, coordination deficits, mouth guard fabrication is to be recommended, and what to do if dentoalveolar trauma occurs, needs to be foretold. Additionally, children with Special Health Care Needs are more likely to be victims of neglect, physical and sexual abuse as compared to the children without disabilities. ${ }^{23}$ Craniofacial injuries are also known to occur in more than half of the cases of child abuse. ${ }^{24}$ Because of these incidences, dentists need to be aware of signs of abuse and the mandatory reporting procedures in such cases. ${ }^{23,24}$

\section{Conclusion}

A patient may deteriorate from his present condition if treatment is not provided because of any limitation due to age, behaviour, inability to cooperate, disability, or medical status. Postponement or neglect of care can result in unnecessary pain, discomfort, increased treatment needs and costs which will increase the patients problem. With unfavourable treatment experiences, and diminished oral health outcomes. Dentists have an obligation to act in an ethical manner in the care of his patients. ${ }^{25}$

"If more dentists knew about the need and took it on a personal level-one that wasn't asking them to change the world, but to just change one life, that alone would make a difference," Rob Saldaña, director of the Denta Cheques program for the National Foundation of Dentistry for the Handicapped (NFDH). ${ }^{6}$

Making a difference in the oral health of a person with special needs may go slowly at first but a strong determination would help bring about positive results for sure. By adopting the strategies discussed in my work above, a very significant impact can be made not only on our patients but on their quality of life as well. Being scared of the dentist is a thing of the past and giving the oral health of such special patients least priority should be forbidden now and forever.

As very correctly said by Mahatma Gandhi that "a customer is the most important visitor on our premises, he is not dependent on us. We are dependent on him. $\mathrm{He}$ is not an interruption in our work. He is the purpose of it. He is not an outsider in our business. He is a part of it. We are not doing him a favour by serving him. He is doing us a favour by giving us an opportunity to serve him" and this is what we all must remember.

I will end by saying that if you focus on treating a disease, you will win or you will lose but if you treat a person, it is for sure that you will win no matter what the outcome may be. So keep on doing a little extra in the service of mankind, for those who require that 'little' more from you. As if we do not lay ourselves in the service of mankind, then whom shall we serve?

\section{References}

1. Commission on Dental Accreditation. Accreditation standards for dental education programs. Chicago: Am Dent Asso 2004.
2. . Waldman HB, Perlman SP. A special care dentistry specialty: sounds good, but... J Dent Educ 2006;70(10):1019-22.

3. American Academy of Pediatric Dentistry. Definition of special health care needs. Pediatr Dent 2012;34(special issue): 16 .

4. Guay AH. Access to dental care. Solving the problem for underserved populations. J Am Dent Assoc 2004; 135(11):1599-605.

5. Davies R, Bedi R, Scully C. Oral health care for patients with special needs. BMJ 2000;321(7259): 495-8.

6. Steinberg BJ. Issues and challenges in special care dentistry. J Dent Educ 2005;69(3):323-4.

7. Serving Patients with Special Needs. Allison M. DiMatteo, BA, MPS

8. Guideline on Management of Dental Patients with Special Health Care Needs. Originating Council Council on Clinical Affairs Review Council Council on Clinical Affairs Adopted 2004 Revised 2008, 2012

9. . American Cleft Palate-Craniofacial Association. Parameters for evaluation and treatment of patients with cleft lip/ palate or other craniofacial anomalies. Chapel Hill, NC: The Maternal and Child Health Bureau, Title V, Social Security Act, Health Resources and Services Administra- tion, US Public Health Service, Dept of Health and Hu- man Services; Revised edition November 2009. Grant \#MCJ-425074.

10. . American Academy of Pediatric Dentistry. Policy on third party reimbursement for oral health care services related to congenital orofacial anomalies. Pediatr Dent 2012;34 (special issue):89-90.

11. . National Foundation for Ectodermal Dysplasias. Parame- ters of oral health care for individuals affected by ecto- dermal dysplasias. National Foundation for Ectodermal Dysplasias. Mascoutah, Ill; 2003.

12. Herdandez P, Ikkanda Z. Applied behavior analysis: Behavior management of children with autism spectrum disorder in dental environments. J Am Dent Assoc 2011;142(3):281-7.

13. US Dept of Health and Human Services. Health Insurance Portability and Accountability Act (HIPAA). Available at: "http://www.aspe.hhs.gov/admnsimp/pl104191. htm". Accessed 24, 2012.

14. American Academy of Pediatric Dentistry. Guideline on record-keeping. Pediatr Dent 2012;34(special issue): 287-94.

15. Charles JM. Dental care in children with developmental disabilities: attention deficit disorder, intellectual disabilities, and autism. J Dent Child 2010;77(2):84-91.

16. US Dept of Justice. Americans with Disabilities Act of 1990, as Amended. Available at: "http://www.ada.gov/ publicat.htm”. Accessed July 4, 2012.

17. American Academy of Pediatric Dentistry. Guideline on informed consent. Pediatr Dent 2012;34(special issue): 295-7.

18. American Academy of Pediatric Dentistry. Guideline on behavior guidance for the pediatric dental patient. Pediatr Dent 2012;34(special issue):170-82.

19. American Academy of Pediatric Dentistry. Policy on dietary recommendations for infants, children, and adolescents. Pediatr Dent 2012;34(special issue):56-8.

20. American Academy of Pediatric Dentistry. Guideline on pediatric restorative dentistry. Pediatr Dent 2012;34 (special issue):214-21.

21. American Academy of Pediatric Dentistry. Guideline on fluoride therapy. Pediatr Dent 2012;34(special issue): $162-5$. 
22. American Academy of Pediatric Dentistry. Policy on interim therapeutic restorations (ITR). Pediatr Dent 2012; 34(special issue):48-9.

23. Giardino AP, Hudson KM, Marsh J. Providing medical evaluations for possible child maltreatment to children with special health care needs, Child Abuse and Neglect 2003;27(10):1179-86.

24. . American Academy of Pediatric Dentistry, American Academy of Pediatrics. Oral and dental aspects of child abuse and neglect. Pediatr Dent 2011;31(special issue): $147-50$.

25. American Academy of Pediatric Dentistry. Policy on the ethical responsibility to treat or refer. Pediatr Dent 2012;34(special issue): 102 . 